

\title{
Reseñas de exposiciones
}

\author{
Manuel Munive Maco \\ Investigador Independiente \\ rupestrecontemporaneo@gmail.com \\ Lima-Perú
}

Las condiciones de vida en las que nos encontramos inmersos desde fines de marzo de este año, imposibilitaron la inauguración de exposiciones de artes visuales y, con ello, el acceso del público a galerías, centros culturales y museos. Para mitigar esta parálisis, surgió una enorme oferta de exposiciones virtuales que han servido para subrayar lo irreemplazable de la experiencia directa con los objetos artísticos, y que su aplicabilidad -mayor aún en el campo educativo- es valiosa si actúa únicamente como complemento de lo presencial.

A continuación, reseñaremos dos de las pocas muestras de artes visuales que se abrieron al público a partir del mes de agosto. La primera, realizada en la sala del Instituto Cultural Peruano Norteamericano, en el centro de Lima y, la segunda, en la filial miraflorina de la uruguaya Galería del Paseo. En el primer espacio se presentaban las obras finalistas y premiadas del concurso de arte contemporáneo que la institución convoca anualmente desde 2018 y, en el segundo, la muestra individual de Nereida Apaza, arequipeña de origen y una de las artistas jóvenes más importantes del Perú. Vale decir que, en ambos casos, las visitas se agendaron mediante correo electrónico y se realizaron siguiendo el protocolo sanitario vigente.

Después de esta experiencia, podemos asegurar que, con un poco de buena voluntad y adaptándonos a los requerimientos del momento, las galerías de arte podrían retomar su funcionamiento sin muchas complicaciones pues, a excepción de las noches inaugurales, nunca están atiborradas de público. Todo lo contrario: lo más frecuente es que las recorramos en soledad, como casi siempre. Y esta circulación de baja intensidad, bien supervisada, podría salvar de la cancelación las programaciones del 2021 e, incluso, retomar aquellos proyectos pospuestos este año. Podrían inaugurarse "virtualmente", por ejemplo, como sucedió con la muestra personal de Apaza, quien "asistió" desde Arequipa, y permanecer abiertas siguiendo un estricto cronograma al que podamos adecuarnos sencillamente. Y esto vale especialmente para las galerías comerciales, cuyos clientes y coleccionistas, lamentablemente, tampoco abundan y deben estar habituados ya a las visitas agendadas.

Esta "nueva normalidad", cuyo límite sigue siendo incierto, no afectará tanto a la actividad del circuito de las artes visuales como lo viene haciendo con el de las artes escénicas, por ejemplo, cuyos operadores se encuentran en verdaderos aprietos por la naturaleza de su vinculación con el público. Lo que tememos los curadores es que algunas instituciones culturales que cuentan con salas de exhibiciones hayan encontrado cierto confort en la inmovilidad determinada por la pandemia y se plieguen a continuarla sin reparos, manteniendo la cancelación de su programa de exhibiciones, descuidando el montaje y el mantenimiento de las mismas, así como abandonando la edición de catálogos y programas de mano impresos, siempre imprescindibles para construir nuestra historia del arte, y hoy más importantes que nunca como documentos urgentes. Debemos estar atentos. 


\section{Premio de Arte Contemporáneo} ICPNA 2020

Si el trabajo que ganó el primer premio de un concurso de artes visuales recae sobre los dos o tres que consideramos los mejores, podemos decir que coincidimos en lo esencial con el fallo del jurado. Sin embargo, existe una diferencia más que abismal entre estas pocas propuestas notables y el nutrido grupo de aquellas que, a pesar de su visible pobreza formal y conceptual, figuran también en el rubro de "finalistas". Esto nos lleva a pensar en la urgencia de corregir las bases del certamen o el proceso de su convocatoria; incluso, tal vez, vigilar la etapa de preselección, es decir, el momento en que se evalúan los proyectos mediante

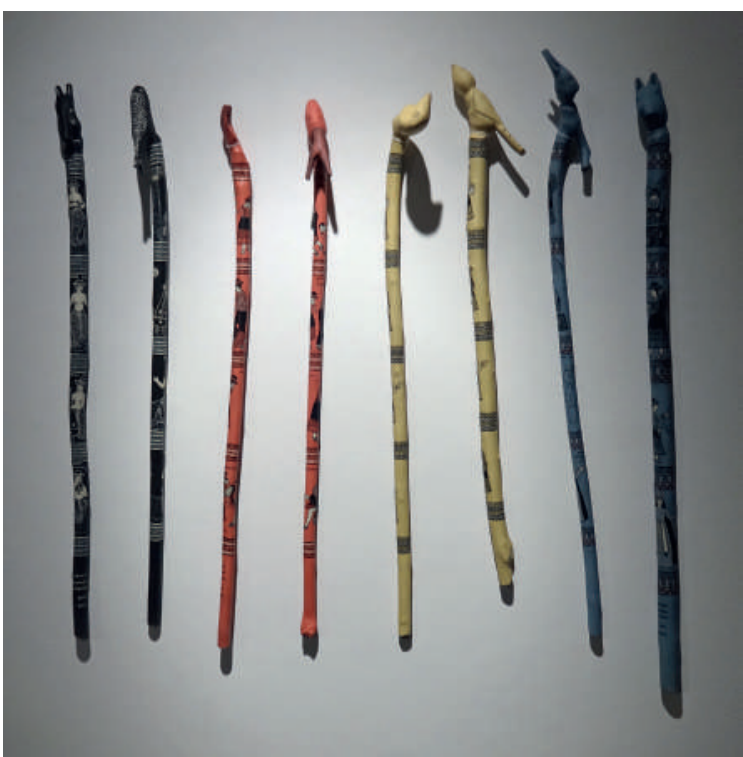

Venuca Evanán, Richary warmi. Bastones decorados, 2019. su documentación. Creemos que allí reside el problema de que, en este concurso de arte, uno de los pocos que se organizan en el Perú, no se deshicieran oportunamente de los proyectos insignificantes: el puñado de fotografías pegadas sobre una cartulina que constituye uno de ellos es el "emblema" de lo que decimos, sin comentar aquellos que, a pesar de su evidente laboriosidad, resultan inolvidables por su infortunio estético.

Entre los cuarenta participantes destacan los nombres de Jorge Cabieses, Edi Hirose, Joaquín Liébana, Santiago Roose, Claudia Salem y Eduardo Villanes, activos en Lima en lo que va del siglo, así como otros cuya trayectoria se ha desarrollado fundamentalmente en el extranjero, como sucede con Marco Pando y Antonio Páucar. La mayoría de ellos, respaldados por varias muestras personales en el Perú y el extranjero, ha obtenido, además, diversos premios y distinciones. Es en la participación de este núcleo de artistas -cuyas edades fluctúan entre los 45 y 50 años- donde radica la importancia simbólica del certamen, el prestigio de sus veredictos y el sentido de su continuidad (No estamos seguros de que la institución esté proyectando enriquecer sus colecciones con estos premios, ya que ese suele ser el fin de un competición semejante, es decir, distinguir a un artista meritorio y adquirir su trabajo para enriquecer un acervo).

Cuando decidimos hacer esta breve reseña tuvimos que regresar a la sala dos semanas después -reserva de por medio- para tomar notas, ya que la institución no produjo ningún material impreso que los espectadores pudiéramos llevarnos. Ha sido desconcertante que una de las exposiciones más importantes de nuestro mermado calendario de actividades, y justo aquella realizada en la sede histórica del ICPNA, no haya dejado hasta hoy -30 de septiembre- ningún documento que los participantes pudieran adjuntar a sus hojas de vida. Es probable que en esta desafortunada decisión de sus directivos haya pesado la proyección de la escasa asistencia de espectadores durante los pocos días y en los acotados horarios que permanece abierta. Parece que no se han percatado de que esa omisión obra en favor del olvido el certamen. Sin duda, ese detalle fue una motivación para redactar estas líneas.

Después de una reflexión resultante del cotejo de los participantes y una rápida pesquisa sobre sus procedencias académicas y formativas, caímos en la cuenta de que lo que refleja 
lo expuesto hoy en la sala del ICPNA es una pugna silente entre dos "bandos": el de los artistas cuyo trabajo es el eslabón de un proceso creativo -de más de veinticinco años en Villanes, o de más de quince en Hirose, por ejemplo-, y el de aquellos que pretenden serlo a partir de propuestas que son ciertamente "ocurrencias" o, en el mejor de los casos, los ejercicios pendientes de una formación académica que experimenta serios problemas (Ante la ausencia de un mercado de arte local para nuestros más jóvenes creadores, parece imponerse la seducción por la experimentación fatua, lo más alejado del rigor que toda pieza que aspira a comercializarse requiere. Desde hace por lo menos quince años la mayoría de quienes se forman como artistas en el Perú saben de antemano que lo más probable es que nunca consigan comercializar su trabajo. Y esta realidad bastaría para que las instituciones formativas replanteen sus objetivos y se pregunten: “¿Estamos formando artistas que subsistirán únicamente gracias al patrimonio de sus familias?”).

La democratización de la experimentación artística que las herramientas tecnológicas han propiciado conllevó también -como es lógico-, a que unos pocos ejercicios deshilvanados basten para que quienes los realizan se autodenominen artistas. Este fenómeno explica la precariedad de muchas de las piezas -video proyecciones, objetos sonoros, fotografías intervenidas, etc.- presentadas al concurso. Pero algo ha pasado también con la indispensable autocrítica del artista. ¿No será que, precisamente, los procesos artísticos, por su naturaleza paulatina, selectiva y depurativa, van conformando esa suerte de "juicio" sobre la propia producción, mientras que las "ocurrencias realizables", por el contrario, buscan el impacto mediante atajos desafortunados? Sin duda, esta efervescencia participativa es una consecuencia inevitable de este tipo de certámenes, y sobre ella debe actuar el jurado, con pinzas, descartando todo aquello que menoscabe la calidad del conjunto; algo que la institución organizadora debería exigir y agradecer.

Pensando en el valor que la trayectoria de un autor adiciona a sus piezas y en el legítimo interés de una institución de premiarlo para prestigiarse a sí misma, elegimos los trabajos de Eduardo Villanes (Moscú, 1967) y Joaquín Liébana (Lima, 1975), como nuestros favoritos. Montesierpe, de Villanes, estaba conformado por una pintura en óleo sobre tela-que incorporaba como materia pictórica la arena del lugar arqueológico cuyo nombre titula la pieza- y un video que registraba el peregrinaje requerido para su ejecución. Desde hace algunos años, los videos que documentan los ejercicios plásticos de Villanes adquieren el valor de piezas autónomas y, en este caso, premiarlo habría significado distinguir un cuadro y una video creación de registro que vincula también las experiencias del land art y la performance. Y en lo que respecta a Liébana, uno de los pocos escultores jóvenes activos $\mathrm{y}$, sin duda, uno de los más prolíficos -junto a Haroldo Higa o Aldo Shiroma, por ejemplo-, y cuyo estilo, encarnado en materiales y tamaños

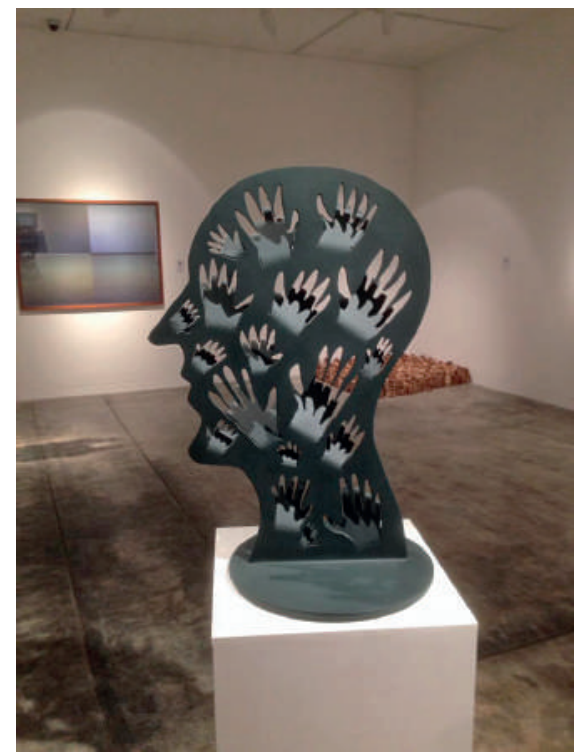

Joaquín Liébana. Pensamientos erróneos. Metal calado, doblado y pintado, 2020.

diversos, es inconfundible, nos pareció merecedor de la distinción. En verdad, la perseverancia de Liébana es encomiable ante el progresivo retraimiento de las propuestas escultóricas auténticas y notables como la suya, una consecuencia, también imprevisible, de la democratización del ejercicio artístico propiciado por los recursos tecnológicos: podríamos 


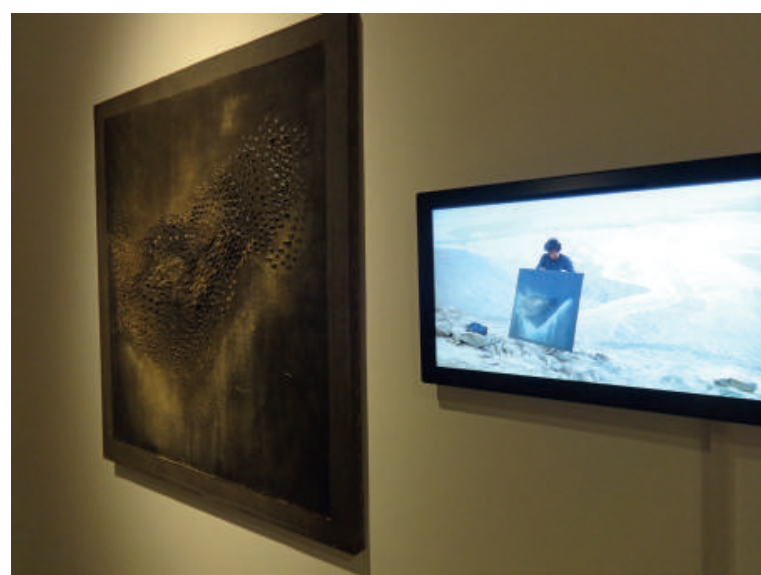

Eduardo Villanes. Montesierpe. Pintura y video, 2017.

decir que la escultura se ha retraído tanto como se ha difundido la fotografía digital.

Es normal que, en una segunda visita a una exposición de arte contemporáneo, individual o colectiva, algunos trabajos se impongan sobre otros, y esto es más patente en el caso de un concurso. Esto pasó con la escultura en hierro calado de Liébana, cuya presencia parecía presidir el amplio sector donde estaba ubicada; también ocurrió con el conjunto de Villanes y con la serie de objetos que componían la participación ganadora. Suponemos que lo mismo

habría pasado con la instalación de Páucar, pero, lamentablemente, había sido retirada para su mantenimiento, según nos informó el personal que custodia la sala (Algo semejante ocurrió con una de las piezas en video -Los campos de fútbol son las áreas verdes más protegidas-, cuyo monitor permaneció apagado durante los sesenta minutos que permanecimos allí. No era un trabajo notable, pero si estaba entre los finalistas debía ser apreciado. Casi nunca se habla del estado de orfandad que en Lima experimentan las piezas artísticas que requieren estar conectadas a un tomacorriente: pueden permanecer apagadas sin que ninguna de las personas a cargo mueva un dedo para que vuelvan a funcionar).

Por suerte, esta tercera edición del concurso del ICPNA será recordada porque consiguió destacar la original y solvente propuesta plástica de una creadora que puede considerarse emergente en la escena del arte local, aunque esté en ejercicio desde hace diecisiete años. El nombre de Venuca Evanán (Lima, 1987), la ganadora del Premio ICPNA 2020, ha resonado desde 2019, cuando obtuvo el Premio Kuna Expressions en la feria Art Lima y resultó finalista del Concurso de Pintura Contemporánea convocado por el Museo del Banco Central de Reserva del Perú. El certamen que ahora comentamos nos permitió ver su trabajo por primera vez, y concluir que no solamente se había premiado una participación notable, sino que, adicionalmente, se estaba incorporando a la dinámica del arte peruano actual una propuesta contemporánea que abreva en la pintura popular andina y, específicamente, en su vertiente moderna, concebida y desarrollada en Lima desde hace unos cuarenta años.

A partir de la breve reseña que Evanán nos proporcionó, colegimos que su formación plástica como artista e ilustradora la realiza trabajando en el círculo familiar, adiestrada por sus padres, Primitivo Evanán y Valeriana Vivanco, célebres pintores de las denominadas tablas de Sarhua, la singularísima expresión plástica de ese distrito ayacuchano, cuya función original de rememorar la genealogía familiar para reforzar sus vínculos, se transforma en una pintura portátil que testimonia y también fortalece a los miembros de su comunidad una vez instalados en Lima. Esta circunstancia problematiza el que la llamemos autodidacta, pues si bien no se formó como pintora durante el lustro que dura normalmente el periodo de estudios en una institución académica, sí lo hizo, y durante mucho más tiempo, bajo la supervisión de dos experimentados maestros.

Así como Villanes incorpora en su propuesta contemporánea la indagación de los procesos de la plástica precolonial y sus motivaciones cultuales -con sus 1700 metros, Montesierpe es posiblemente el geoglifo figurativo precolonial más grande del Perú-, Evanán trae consigo 
un proceso cultural andino, más breve sin duda -la más antigua de las tablas de Sarhua data de 1876, según Macera, quien recomienda denominarlas "vigas"-, pero imbricado irreversiblemente con el fenómeno de la migración y la mentalidad andina que prevalece en el contexto urbano. Pero por encima de esta filiación cultural, el trabajo de Evanán -conformado por ochos bastones pintados con empuñaduras talladas- resulta una auténtica revelación como espécimen al arte contemporáneo peruano. El trabajo de las figuras pintadas, tanto su anatomía como su indumentaria, es sobresaliente.

Lamentablemente, esta serie de bastones o cayados requería de un montaje imaginativo que facilitara la apreciación cabal de sus detalles y subrayara el acierto de su distinción. Colgarlos sobre el muro, a pesar de que llevan diversas figuras femeninas pintadas a lo largo de su fuste -vírgenes, sirenas, mujeres empoderadas y mujeres violentadas- ni siquiera facilita el registro fotográfico. Nos parece que lo mejor habría sido presentarlos hincados sobre un pedestal que pudiéramos rodear. La singularidad formal y simbólica de esta serie de varas talladas y pintadas merecía de una disposición esmerada. ¿Hasta qué punto la supervisión de la museografía de las piezas seleccionadas en un concurso le compete al jurado? (Incluso la participación de la escultora Claudia Salem, compuesta por dos estructuras reticulares de acero incrustadas sobre el muro, las cuales configuraban dos siluetas humanas, estaba en el lugar equivocado: la escasa altura de la sala del primer piso la oprimía, lo que no habría sucedido en el mucho más alto salón del sótano, donde incluso el efecto de su sombra se habría apreciado mejor).

El triunfo de Venuca Evanán resulta significativo, también, porque incorpora los instrumentos de la pintura de tablas de Sarhua, fundamentalmente, tierras de color y plumas de ave, a la ya amplia panoplia de materiales con los que un artista peruano cuenta para elaborar una pieza contemporánea.

\section{LA LUZ DE LA OSCURIDAD NEREIDA APAZA \\ Galería del Paseo}

Así como celebramos el acierto del jurado en un concurso de arte, aplaudimos también que una artista cuyo trabajo admiramos consiga comercializarlo. Y esto ha sucedido recientemente con la obra de Nereida Apaza, aun cuando su valía ha sido largamente refrendada. Apaza obtuvo, por ejemplo, el Segundo Puesto del 10. Concurso "Pasaporte para un Artista" (2007); el Primer Premio del Salón Nacional de Acuarela del Icpna (2010) y el Primer Premio del Concurso Nacional de Pintura del Banco Central de Reserva del Perú (2012), sin contar las numerosas veces que ha resultado finalista en

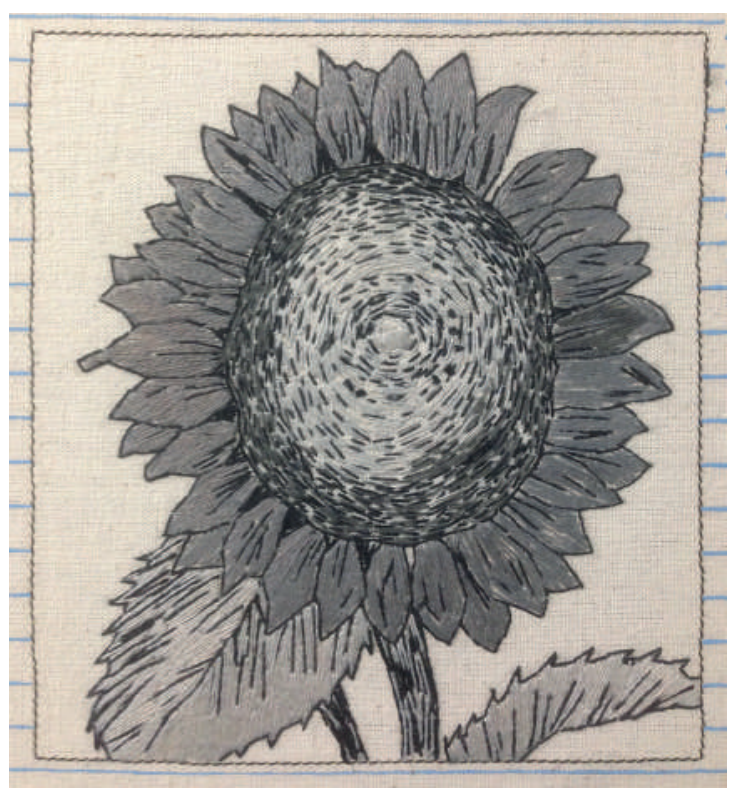

Nereida Apaza. Pormenor de bordado sobre tela, 2020. 
otros certámenes nacionales. ${ }^{1}$ Sin embargo, todas estas distinciones no pesaron, aparentemente, para que las galerías comerciales y los coleccionistas de Lima la descubrieran, algo que ha ocurrido -por fin- durante el último año. Tampoco parece haber influido a su favor el reconocimiento que algunos críticos le han expresado desde hace tiempo, ni el que algunos curadores la hayan incorporado a sus proyectos o, incluso, que

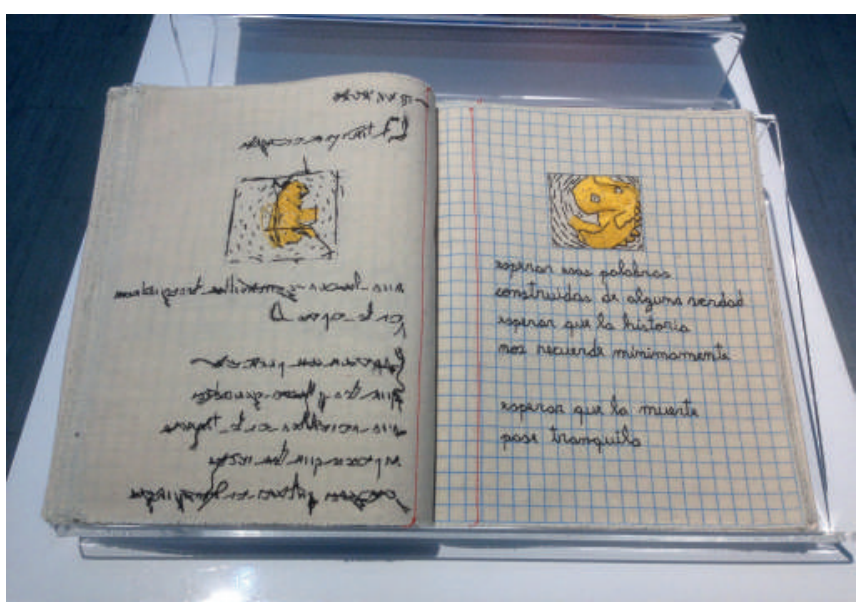

Nereida Apaza. Poemario bordado, 2020. esté representada en las colecciones de algunos museos públicos y, mucho menos, que sean sobre todo los artistas más jóvenes quienes la ven como un referente. Basta este solo caso para que comprendamos la urgencia de generar un mercado de arte para los jóvenes creadores del Perú y, particularmente, para aquellos que, como ella, viven y trabajan fuera de Lima, es decir, en terra ignota para muchos de nuestros galeristas y coleccionistas. ${ }^{2}$

La luz de la oscuridad fue el título de la duodécima muestra individual que Nereida Apaza (Arequipa, 1979) acaba de clausurar en la Galería del Paseo, la segunda en Lima después de diez años y la primera en un espacio comercial. ${ }^{3}$ Esta relación entre la artista y la galería se consolidó a fines de 2019, cuando participó en una de sus muestras colectivas, justo en la víspera del vuelo que la llevaría a Londres para realizar una residencia artística. Al parecer, en el Museo Británico había personas que estaban al tanto de su trayectoria, y se mostraron particularmente interesadas en aquellas curiosas piezas que, por su naturaleza textil definida por el bordado, eran simultáneamente contemporáneas y potencialmente "museables". En efecto, el objetivo de esa residencia consistía, básicamente, en que Nereida elaborase una propuesta artística generada a partir del contacto o el "diálogo" -visual y táctil- con los textiles precolombinos y tradicionales peruanos de sus colecciones, la cual se expondría brevemente allí, lo que ocurrió en el mes de diciembre del mismo año.

Y, ¿desde cuándo Nereida Apaza se sirve del bordado para elaborar su obra artística? En el catálogo de su sexta muestra individual -El corazón de un pájaro, inaugurada en la sala de El Cultural de Arequipa en 2010-, los bordados aparecen ya como un recurso al lado de la acuarela, la serigrafía, el collage, la técnica mixta y el libro-objeto. ${ }^{4}$ Pero también, desde entonces, la artista asedia, recreándolo, ese espacio en el que los peruanos pasamos

1 Y el año pasado, su cortometraje de animación, Historia Secreta, figuró entre los premiados en el concurso de obras experimentales de la DAFO.

2 Habría que ampliar la mirada sobre la dinámica cultural de otras ciudades del país, pero eso parece imposible: la reciente convocatoria al Premio Bicentenario, por ejemplo, no hará otra cosa que premiar un proyecto curatorial nacional que se realizará en dos sedes de Lima. ¿Es que las autoridades que lo organizan jamás escucharon de la necesidad de combatir el centralismo limeño? Esperemos que sí lo comprendan quienes se hagan cargo de las conmemoraciones del Tricentenario.

3 La anterior se realizó en la sala del Icpna de Lima en 2010, es decir, en un espacio dedicado exclusivamente a la difusión cultural.

4 Ese mismo año hace una de sus primeras experimentaciones en video -Las parcas- y realiza Dialéctica de la paz, una intervención en la Plaza de Armas que ella consigna, además, como su cuarta individual. 
confinados once años de nuestras vidas: el aula escolar y su sempiterna utilería -carpetas, pizarrón, mapa político-y, muy especialmente, los pequeños cuadernos sobre cuyas pautadas páginas, azorados, tratábamos de aprehender el mundo. ${ }^{5}$ Creo que esa experiencia compartida por todos -o casi todos- explica la fascinación que el público experimenta ante este objeto insólito y entrañable creado por Nereida, el cual se ha convertido en la pieza protagónica de sus exhibiciones personales y colectivas. El cuaderno, al igual que el libro, "atiende" persona a persona, nos atrae por su escala y, en este caso, por la lectura de su esmerada escritura "Palmer" que, como una voz pueril, conduce nuestra visita. Desde luego que existen en nuestro medio, artistas notables y de varias generaciones que han hecho de la aguja y el hilo sus instrumentos primordiales, pero los cuadernos de dibujos y escritura bordados de Apaza parecen no tener parangón.

Y esta obra construida con aguja e hilo que interesó a los curadores del Museo Británico en Londres y a la galerista uruguaya que dirige la Galería del Paseo, ha servido para que el abotagado mercado de arte limeño reaccionara un poco ante el trabajo de la artista arequipeña. Este "descubrimiento" ha hecho que circulen incluso las piezas elaboradas muchos años atrás, las cuales, gracias a la exigencia técnica de la autora, se encuentran en perfecto estado de conservación y ahora repotenciadas como eslabones sustanciales de su singular proceso creativo.

Pero esta exposición tuvo una virtud adicional: fue elaborada íntegramente durante el periodo de emergencia sanitaria, el cual fue particularmente severo en Arequipa. Si bien este lapso puede parecer larguísimo para quienes llevan una vida regida por la rutina de la "antigua normalidad", resulta extremadamente corto para preparar toda una muestra personal. Sin embargo, la artista, como si se tratase de conjurar la adversidad, trabajó intensamente y culminó varias series de piezas bordadas, desde cuadernos que son poemarios ilustrados hasta páginas sueltas articuladas como polípticos, entre las que destacan sus herbarios poéticos -los grupos titulados Primera intuición y Semillas-, cuyas flores parecen dibujos en "línea valorada", así como los novedosos planos de ciudades imaginarias cuya cuadrícula urbana configura una palabra que debemos encontrar y que resuena como una declaración colectiva. La escritura poética de la artista, de la cual habría que tratar específicamente en otra ocasión, la salvaguardó de incurrir en obviedades y lugares comunes. En verdad, el yo poético que enuncian sus textos bordados transmite la calma de quien sabe que la vida termina por imponerse, pese a todo. (Únicamente algunas pistas -como la dedicatoria a Cecilia Capira, por ejemplo- nos devolvían al agobiado presente).

Nunca antes, como durante la solitaria visita a la galería -con tapabocas y todo-, fuimos más conscientes de que algo aparentemente tan simple como asistir a una exposición implicaba una peregrinación y un "retiro" momentáneo, esto es, buscar en soledad la experiencia estética allí donde se halle. Tampoco pudimos eludir la permanente imagen de Nereida Apaza trabajando a mano y en la máquina de coser de su madre -de quien aprendió el oficio de bordar-, esos trabajos que nos reconcilian con la infancia y reivindican la escuela, y entre los que comparece, discretamente como siempre, el profesor Teodoro Apaza Flores, su papá.

5 Entre las piezas de Apaza en las que resulta determinante la evocación de la formación escolar peruana destacamos Primer grado, instalación de 2010; Historia general, políptico de 2011 que forma parte de la colección del Museo de Arte de la Universidad Nacional Mayor de San Marcos; Dictado, políptico de 2012 con el que obtuvo el Primer Premio del Concurso del Banco Central de Reserva, y La utopía de la educación, instalación que formó parte de su más reciente muestra personal en El Cultural de Arequipa en julio de 2019. 